\title{
BMJ Open SPECT V/Q for the diagnosis of pulmonary embolism: protocol for a systematic review and meta-analysis of diagnostic accuracy and clinical outcome
}

\author{
Pierre-Yves Le Roux, ${ }^{1}$ Philippe Robin, ${ }^{1,2}$ Cécile Tromeur, ${ }^{3}$ Alexandra Davis, ${ }^{4}$ \\ Helia Robert-Ebadi, ${ }^{5}$ Marc Carrier, ${ }^{2}$ Francis Couturaud, ${ }^{3}$ Grégoire Le Gal, ${ }^{2,3}$ \\ Pierre-Yves Salaun ${ }^{1}$
}

To cite: Le Roux P-Y, Robin P, Tromeur C, et al. SPECT V/Q for the diagnosis of pulmonary embolism: protocol for a systematic review and metaanalysis of diagnostic accuracy and clinical outcome. BMJ Open 2018;8:e022024. doi:10.1136/ bmjopen-2018-022024

- Prepublication history and additional material for this paper are available online. To view these files, please visit the journal online (http://dx.doi. org/10.1136/bmjopen-2018022024).

Received 31 January 2018 Revised 15 March 2018 Accepted 3 April 2018

Check for updates

${ }^{1}$ Service de médecine nucléaire, CHRU de Brest, EA3878 (GETBO), Université de Brest, Brest, France

${ }^{2}$ Department of Medicine, Ottawa Hospital Research Institute, University of Ottawa, Ottawa, Ontario, Canada ${ }^{3}$ Département de Médecine Interne et Pneumologie, CHRU de Brest, EA3878 (GETBO), Université de Brest, Brest,

France

${ }^{4}$ Library and Learning Centre, The Ottawa Hospital, Ottawa, Ontario, Canada

${ }^{5}$ Division of Angiology and Hemostasis, Geneva University Hospital and Faculty of Medicine, Geneva, Switzerland

Correspondence to

Professor Pierre-Yves Le Roux; pierre-yves.leroux@chu-brest.fr

\section{ABSTRACT}

Introduction Single photon emission computed tomography ventilation/perfusion (SPECT V/Q) imaging has many proponents within the nuclear medicine community and has already largely replaced planar $\mathrm{V} / \mathrm{Q}$ scintigraphy in daily practice for the diagnosis of pulmonary embolism (PE). However, the test is still described in clinical guidelines as an experimental test because of insufficient evidence.

Methods and analysis We will conduct a systematic review and a meta-analysis of diagnostic accuracy and management outcome studies involving patients evaluated with V/Q SPECT for suspected acute PE. We will search from inception to 19 December 2017 MEDLINE, Embase and the Cochrane Central Register of Controlled Trials for diagnostic accuracy studies, randomised controlled trials and observational cohort studies. Two reviewers will conduct all screening and data collection independently. The methodological quality and risk of bias of eligible studies will be carefully and rigorously assessed using the Quality Assessment of Diagnostic Accuracy Studies-2, the Cochrane Collaboration's tool and the Risk Of Bias In Non-randomised Studies - of Interventions (ROBINS-I) tools. The primary outcomes will be sensitivity, specificity and likelihood ratios of V/Q SPECT for the diagnosis of acute PE. The secondary outcomes will be the rate of venous thromboembolism during a 3 -month follow-up period in patients left untreated after a negative diagnostic work-up based on SPECT V/Q. Ethics and dissemination This study of secondary data does not require ethics approval. It will be presented internationally and published in the peerreviewed literature.

PROSPERO registration number CRD42018084095.

\section{INTRODUCTION}

Rationale

The diagnosis of pulmonary embolism $(\mathrm{PE})$ remains challenging. Both missed diagnosis and excess diagnosis have undesirable consequences. Undiagnosed PE could be fatal in up to $25 \%$ of patients ${ }^{1}$
Strengths and limitations of this study

- Although single photon emission computed tomography ventilation/perfusion (SPECT V/Q) has already been largely implemented in daily practice for $\mathrm{PE}$ diagnosis, the test is still described as experimental in clinical guidelines.

- Many studies have been published on SPECT V/Q for pulmonary embolism (PE) diagnosis but the performance of the test is still unclear because of methodological issues.

- The performances of SPECT V/Q for the diagnosis of PE will be assessed using objective and widely accepted tools for quality assessment.

- This systematic review and meta-analysis should provide elements of responses to the ongoing debate about the evidence of SPECT V/Q for the diagnosis of acute PE.

while anticoagulant therapy carries a risk of bleeding. ${ }^{2}$ Moreover, there is a trend towards indefinite duration of anticoagulant therapy after a first episode of PE that was not provoked by a major risk factor. ${ }^{3} \mathrm{~A}$ definitive and accurate diagnostic conclusion is therefore required for all patients with suspected PE.

The diagnostic strategy starts with clinical probability assessment and D-dimer testing. In patients with a non-high/unlikely clinical probability but positive D-dimer, and in patients with a high/likely clinical probability, lung imaging is required. CT pulmonary angiography (CTPA) is currently the predominantly used imaging test for PE. The test has been widely validated in diagnostic accuracy and management outcome studies. ${ }^{4-6}$ However, CTPA has some limitations, including the use of an iodinated contrast agent, approximatively $5 \%$ of inconclusive tests, higher amount of 
radiation to the breasts compared with alternative tests and an increasing concern regarding a possible overdiagnosis and overtreatment of PE. ${ }^{78}$

The alternative for PE diagnosis is lung scintigraphy. Planar ventilation/perfusion $(\mathrm{V} / \mathrm{Q})$ scintigraphy is also a well-established test for the diagnosis of PE. ${ }^{9}$ The accuracy of the test was assessed in the Prospective Investigation of Pulmonary Embolism Diagnosis (PIOPED) study, which is the planar $\mathrm{V} / \mathrm{Q}$ scan landmark accuracy study versus pulmonary angiography. ${ }^{10}$ Diagnostic strategies based on planar $\mathrm{V} / \mathrm{Q}$ scan were then widely validated in large management outcome studies. ${ }^{811-13}$

In recent years, the technology around $\mathrm{V} / \mathrm{Q}$ scintigraphy has rapidly evolved allowing the introduction of single photon emission computed tomography (SPECT), a new method of scintigraphic acquisition. ${ }^{14}$ SPECT V/Q has been reported to improve the diagnostic performances of the test and significantly decrease the proportion of non-diagnostic studies. ${ }^{1516}$ The Nuclear Medicine community is mostly enthusiastic and, already back in 2009, the European Association of Nuclear Medicine guidelines for $\mathrm{V} / \mathrm{Q}$ scintigraphy strongly recommended the use V/Q SPECT over planar for PE diagnosis. ${ }^{17}$ Consequently, V/Q SPECT has already largely replaced V/Q planar scintigraphy in many nuclear medicine departments. In a survey performed in 2015 among 331 institutions from Australia, Canada and France, more than 2/3 of centres performed SPECT rather than planar images for PE diagnosis. ${ }^{18}$

Many accuracy studies aimed at assessing the diagnostic accuracy of SPECT for PE diagnosis. More recently, a few systematic reviews and meta-analyses have been published, ${ }^{19-21}$ and concluded that SPECT was superior to planar imaging, ${ }^{19}$ and superior ${ }^{21}$ or equal to CTPA. ${ }^{19}$ However, the clinical community remains much more cautious about the use SPECT V/Q for PE diagnosis. In particular, the specialists in venous thromboembolism (VTE) consistently describe SPECT V/Q as an experimental test rather than as an established imaging modality. ${ }^{7922} 23$ Indeed, the exact accuracy of the test is still unclear because of methodological issues. For example, in many of available accuracy studies, there is an incorporation bias with the V/Q SPECT result participating in the final diagnostic conclusion. It is important to note that the previously published systematic reviews and meta-analyses on the diagnostic accuracy of SPECT have incorporated these studies with an incorporation bias into the analyses. This represents an important limitation and needs to be encounted for in the interpretation of the pooled results. In addition, there is wide heterogeneity within the studies in terms of reference standard, acquisition protocol and criteria used for interpretation. Finally, although some studies reported on the risk of VTE during follow-up in patients in whom the diagnosis of $\mathrm{PE}$ was ruled out on the basis of a negative V/QSPECT, most did not use a standardised algorithm and additional tests were often performed at the discretion of the physicians. Thus, the clinical outcome of patients investigated with $\mathrm{V} / \mathrm{Q}$ SPECT remains unclear.
Given this paradoxical situation of a test already largely implemented in daily practice but still described as experimental by clinicians, there is an urgent need to perform a systematic review on the performances of SPECT V/Q for the diagnosis of $\mathrm{PE}$, using objective and widely accepted tools for quality assessment.

\section{Objectives}

The primary objective of this systematic review and meta-analysis is to establish the diagnostic accuracy (sensitivity, specificity, likelihood ratios (LRs)) of SPECT V/Q for the diagnosis of acute PE.

The secondary aim is to review the clinical outcomes of patients investigated for suspicion of acute PE with a standardised algorithm based on V/Q SPECT.

\section{METHODS}

This protocol followed the recommendations in the statement on Preferred Reporting Items for Systematic Reviews and Meta-Analyses Protocol (PRISMA-P) ${ }^{24}$ and PRISMA of Diagnostic Test Accuracy (PRISMA-DTA). ${ }^{25}$ This protocol has been published in the PROSPERO International Prospective Register of Systematic Reviews with registration number CRD42018084095.

\section{Eligibility criteria}

Studies will be selected according to the criteria outlined below.

\section{Study designs}

To answer to the primary objective (diagnostic accuracy), we will include diagnostic accuracy studies of $>10$ patients.

To answer to the secondary objective (clinical outcome), we will include randomised controlled trials (RCTs) and prospective cohort studies. Studies with other types of designs, including retrospective cohort studies, casecontrol studies, case series and case reports will be excluded.

\section{Participants/Interventions}

The study population of interest will include adults (aged 18 years or older) with a suspected acute PE investigated using a SPECT V/Q.

\section{Comparator}

This study will be non-comparative.

\section{Outcome}

The primary outcomes will be sensitivity, specificity and positive and negative LRs of V/Q SPECT for the diagnosis of acute PE.

The secondary outcomes will be:

- The rate of VTE during a 3-month follow-up period in patients left untreated after a negative diagnostic work-up based on SPECT V/Q.

- The percentage of patients anticoagulated based on a positive $\mathrm{V} / \mathrm{Q}$ SPECT study among patients assessed with $\mathrm{V} / \mathrm{Q}$ SPECT for suspected acute PE. 


\section{Information sources and search strategy}

The following databases will be searched during the electronic component of the systematic review: Medline (via OVID), Embase Classic+Embase (via OVID) and Cochrane's Central Register of Controlled Trials. The specific search strategies will be created by a Health Sciences Librarian with expertise in the design of systematic review searching. A search strategy will be developed to define subject headings and keywords for all searches (see online supplementary appendix 1 for Medline search). After the Medline strategy is finalised, it will be adapted to the syntax of the other databases. There will be no beginning date identified, while the cut-off date will be 19 December 2017. There will be no language exclusion criteria nor any other publication restrictions. Additional references will be sought by hand-searching the bibliographies of relevant articles.

\section{Study records}

Data management

Literature search results will be imported into EndNote, de-duplicated and then uploaded to the Covidence platform (www.covidence.org) to facilitate collaboration among the reviewers during the study selection process.

\section{Selection process}

Two reviewers will independently conduct title and abstract screening procedures. Full reports for all titles and abstracts that appear to meet the inclusion criteria or where there is any uncertainty will be obtained. Conflicts in screening will be resolved by consensus or by a third person. Search results and study selection will be illustrated in a PRISMA flow diagram, ${ }^{26}$ with reasons specified for excluding articles during full-text screening.

\section{Data collection process}

A structured data extraction form will be developed to facilitate the process of data collection from the studies that have been included in the review. Two reviewers will independently extract all data. Discrepancies between reviewers will be resolved by consensus or by a third person if necessary.

\section{Data items}

We will extract:

- Study information: reference ID, authors, year of publication, journal and publication status.

- Study characteristics: country, study period, funding, prospective or retrospective, single centre or multicentre.

- Population characteristics: age, gender, sample size, referral pattern (eg, outpatients, emergency room or inpatients), prevalence of $\mathrm{PE}$, number of participants lost to follow-up.

For accuracy studies:

- Patient selection: consecutive or random sample, inclusion and exclusion criteria, clinical probability for PE, additional testing including D-dimer, intended use of the index test, clinical setting.
- Information related to the index test, that is, $\mathrm{V} / \mathrm{Q}$ SPECT: radiopharmaceuticals, combined CT acquisition, interpretation criteria (positivity thresholds), blinding information, number of readers and resolution of disagreements.

- Information related to the reference standard: imaging procedures, interpretation method, follow-up.

- Flow and timing: time interval and any interventions between the index test and reference standard, patients who did not receive the index test and/or reference standard, patients excluded from the $2 \times 2$ table.

- Outcomes to estimate the test accuracy in the form of a cross tabulation of index test with reference standard ( $2 \times 2$ table) including the number of true positives, false positives, false negatives and true negatives.

For outcome studies:

- Patient selection: consecutive or random sample, inclusion and exclusion criteria, clinical probability for PE, additional testing including D-dimer.

- Study design: RCT or prospective cohort study, a priori defined standardised diagnostic strategy to confirm or exclude PE, blind interpretation of the V/Q SPECT, length of follow-up.

- Information related to $\mathrm{V} / \mathrm{Q}$ SPECT: radiopharmaceuticals, combined CT acquisition, interpretation criteria (positivity thresholds), interpretation method, number of negative, positive and indeterminate results.

- Information related to the diagnostic strategy and therapeutic management: additional testing (D-dimer test, compression ultrasound (CUS), CTPA or V/Q planar) performed in addition to the $\mathrm{V} / \mathrm{Q}$ SPECT.

- Outcomes: number of patients with a positive and a negative diagnostic work-up with regard to PE diagnosis, number of patients who received anticoagulation, number of patients lost to follow up, number of patients eligible for outcome assessment, that is, patients left untreated after a negative V/Q SPECT with a 3-month follow-up, number of thromboembolism events during the 3-month follow-up period in patients left untreated, number and cause of death.

\section{Outcomes and prioritisation}

The primary outcomes will be sensitivity, specificity and negative and positive LRs of V/Q SPECT for the diagnosis of acute $\mathrm{PE}$.

The secondary outcome will be the rate of VTE during a 3-month follow-up period in patients left untreated after a negative diagnostic work-up.

\section{Risk of bias of individual studies}

The methodological quality and risk of bias of eligible studies will be carefully and rigorously assessed. The risk of bias for each study will be ascertained by two reviewers. Results will be compared and disagreements resolved by discussion or, if needed, with the help of a third reviewer. 
Table 1 QUADAS-2 tool to evaluate the risk of bias and concern about applicability of diagnostic accuracy studies

\begin{tabular}{|c|c|c|c|c|}
\hline Domain & Patient selection & Index test & $\begin{array}{l}\text { Reference } \\
\text { standard }\end{array}$ & Flow and timing \\
\hline Description & $\begin{array}{l}\text { Describe methods } \\
\text { of patient selection: } \\
\text { Describe included } \\
\text { patients (prior testing, } \\
\text { presentation, intended } \\
\text { use of index test and } \\
\text { setting): }\end{array}$ & $\begin{array}{l}\text { Describe the index } \\
\text { test and how it was } \\
\text { conducted and } \\
\text { interpreted: }\end{array}$ & $\begin{array}{l}\text { Describe the } \\
\text { reference standard } \\
\text { and how it was } \\
\text { conducted and } \\
\text { interpreted: }\end{array}$ & $\begin{array}{l}\text { Describe any patients who did } \\
\text { not receive the index test(s) and/ } \\
\text { or reference standard or who were } \\
\text { excluded from the } 2 \times 2 \text { table (refer } \\
\text { to flow diagram): Describe the } \\
\text { time interval and any interventions } \\
\text { between index test(s) and } \\
\text { reference standard: }\end{array}$ \\
\hline $\begin{array}{l}\text { Signalling } \\
\text { questions } \\
\text { (yes/no/unclear) }\end{array}$ & $\begin{array}{l}\text { Was a consecutive } \\
\text { or random sample of } \\
\text { patients enrolled? }\end{array}$ & $\begin{array}{l}\text { Were the index test } \\
\text { results interpreted } \\
\text { without knowledge } \\
\text { of the results of the } \\
\text { reference standard? }\end{array}$ & $\begin{array}{l}\text { Is the reference } \\
\text { standard likely to } \\
\text { correctly classify the } \\
\text { target condition? }\end{array}$ & $\begin{array}{l}\text { Was there an appropriate interval } \\
\text { between index test(s) and } \\
\text { reference standard? }\end{array}$ \\
\hline
\end{tabular}

Was a case-control design avoided?

Did all patients receive a reference standard?

standard results interpreted without knowledge of the results of the index test?

If a threshold was used was it prespecified?

Did the study avoid inappropriate exclusions?

Did all patients receive the same reference standard?

Were all patients included in the analysis?

\begin{tabular}{|c|c|c|c|c|}
\hline $\begin{array}{l}\text { Risk of bias: High/ } \\
\text { low/unclear }\end{array}$ & $\begin{array}{l}\text { Could the selection } \\
\text { of patients have } \\
\text { introduced bias? }\end{array}$ & $\begin{array}{l}\text { Could the conduct } \\
\text { or interpretation of } \\
\text { the index test have } \\
\text { introduced bias? }\end{array}$ & $\begin{array}{l}\text { Could the reference } \\
\text { standard, its } \\
\text { conduct or its } \\
\text { interpretation have } \\
\text { introduced bias? }\end{array}$ & $\begin{array}{l}\text { Could the patient flow have } \\
\text { introduced bias? }\end{array}$ \\
\hline $\begin{array}{l}\text { Concerns } \\
\text { regarding } \\
\text { applicability: High/ } \\
\text { low/unclear }\end{array}$ & $\begin{array}{l}\text { Are there concerns } \\
\text { that the included } \\
\text { patients do not match } \\
\text { the review question? }\end{array}$ & $\begin{array}{l}\text { Are there concerns } \\
\text { that the index test, its } \\
\text { conduct or interpretation } \\
\text { differ from the review } \\
\text { question? }\end{array}$ & $\begin{array}{l}\text { Are there concerns } \\
\text { that the target } \\
\text { condition as defined } \\
\text { by the reference } \\
\text { standard does not } \\
\text { match the review } \\
\text { question? }\end{array}$ & \\
\hline
\end{tabular}

\section{Quality assessment}

Diagnostic accuracy

The Quality Assessment of Diagnostic Accuracy Studies (QUADAS)-2 tool will be used to evaluate the risk of bias and concern about applicability of primary diagnostic accuracy studies $^{27}$ (see table 1 ).

We will consider appropriate the following reference standard tests: negative D-dimer in the presence of low or intermediate or unlikely clinical probability, planar $\mathrm{V} / \mathrm{Q}$ scan when used and interpreted according to well-accepted diagnostic algorithms, CTPA or pulmonary angiography. Special attention will be paid to the independence of the reference standard with regard to the V/Q SPECT results in order to avoid any incorporation bias, and to the blind interpretation of the $\mathrm{V} / \mathrm{Q}$ SPECT. The description of the reference standard will be considered appropriate if explained with sufficient detail to permit replication of the test. The time between reference standard and index test will be considered acceptable if $<24$ hour.

A judgement as to the possible risk of bias and concern about applicability on each item in the domains will be made. The results of the QUADAS-2 assessment for all included studies will be summarised in a table and a graphical display. If the answers to all signalling questions for a domain are 'yes', then the risk of bias and concern regarding applicability will we judged low. If a study is judged 'high' or 'unclear' in one or more domains, then it will be judged 'at risk of bias' or as having 'concerns regarding applicability.'

\section{Clinical outcomes}

RCTs will be appraised using the Cochrane Collaboration's tool for assessing risk of bias. ${ }^{28}$ For studies that have used a cohort design, the Risk Of Bias In Non-randomised 
Studies - of Interventions (ROBINS-I) tool ${ }^{29}$ for assessing the quality of non-randomised studies in meta-analyses will be used.

Particular attention will be paid to several criteria: (1) prospective enrolment of unselected patients with suspected PE; (2) a standardised PE diagnostic algorithm and management. If performed, the use of additional testing such as D-dimer test, lower-limb venous CUS or CTPA should be standardised and not left at the discretion of the attending physicians. Standardised management is necessary to permit generalisation of results; and (3) reliability of the 3-month follow-up (completeness, description of reasons for incomplete follow-up) and outcome measures (definition at the start of the study, objectivity).

\section{Data synthesis}

\section{Diagnostic accuracy}

The first step in data synthesis will consist in calculating the descriptive statistics in each primary study. These statistics will include sensitivity, specificity, positive LR and negative LR from the diagnostic $2 \times 2$ tables of primary studies. Using the calculated descriptive statistics with the 95\% CI of each of the primary studies, a descriptive forest plot will be derived. A summary receiver operating characteristic plot will also be developed.

The main objective of this systematic review is to combine data from primary accuracy studies on $\mathrm{V} / \mathrm{Q}$ SPECT for the diagnosis of acute PE. Prior to pooling results, the research team will also assess studies for clinical and methodological heterogeneity through comparison of important study characteristics including those related to the study design, patients, V/Q SPECT acquisition and interpretation (positivity threshold). The degree of statistical heterogeneity will be measured and interpreted using a combination of Cochrane's $Q$ (statistically significant at $\mathrm{P}<0.10)$ and the $\mathrm{I}^{2}$ statistic $(>50 \%$ considered substantial). An $\mathrm{I}^{2}$ value $>75 \%$ is indicative of a very high degree of heterogeneity and, if encountered, the data will not be pooled. A bivariate random-effects regression approach will be used for summary estimates of both sensitivity and specificity.

\section{Clinical outcome}

The second objective of this systematic review is to combine data from management outcome studies to assess the safety of a diagnostic strategy based on $\mathrm{V} / \mathrm{Q}$ SPECT to rule out acute PE. This will be assessed by pooling the rate of VTE event during a 3-month follow-up period in patients left untreated after a negative diagnostic work-up based on SPECT V/Q. Only high-quality studies will be included in the meta analysis, that is, studies with (1) prospective enrolment of unselected patients with suspected PE; (2) an a priori defined PE diagnostic algorithm and management; and (3) a reliable 3-month follow-up and primary outcome measure. Prior to pooling results, the research team will also assess studies for clinical and methodological heterogeneity as described above. If homogeneity among studies is judged as satisfactory, then the results from trials will be pooled using standard meta-analysis procedures.

\section{Patient and public involvement}

Patients and public will not be involved in this study since this is a systematic review on published studies.

\section{DISCUSSION}

In this systematic review and meta-analysis, we aim to assess the diagnostic performance of SPECT V/Q for the diagnosis of acute PE. We decided to assess both diagnostic accuracy of the test and the clinical outcome of patients during follow-up. Studies of new diagnostic tests for PE fall into two broad categories that correspond to early and late stages of test evaluation: accuracy and outcome studies. Accuracy studies determine the accuracy of a new test by comparing the findings with an established 'gold' or reference standard. In such a study, the diagnostic conclusion is based only on the results of the gold standard test. The new test is blindly read, which allows an independent determination of accuracy indices and the assessment of the optimal diagnostic cut-off for quantitative variables. Outcome studies assess the safety and usefulness of the test used the actual diagnostic management of patients. The new test becomes decisional, that is, the diagnostic conclusion and the therapeutic decision are made on the basis of the test's result. The main evaluation criterion is the safety of using the test in deciding patient management and treatment. For VTE diagnosis, a widely accepted criterion is the 3-month rate of VTE in patients left without anticoagulant treatment after a negative strategy. A diagnostic strategy is deemed to safely exclude PE when the 3-month risk is not higher than $3 \%$. This 3\% rate corresponds to the upper limit of the $95 \%$ CI of VTE rate observed in patients with suspected PE left without anticoagulation after a negative pulmonary angiography. ${ }^{30}$

\section{Limitations and challenges}

In many available studies, the SPECT $\mathrm{V} / \mathrm{Q}$ results were provided to the clinicians at the time of the diagnostic work up, and hence participated in the diagnostic conclusion. Therefore, the index test became part of the 'reference standard'. This obviously introduces a major bias and artificially improves the accuracy of the test. Previously published meta-analysis did include such studies in their pooled accuracy indices estimates. ${ }^{19-21}$ We will pay particular attention to the independence and blind interpretation between the index test and the reference standard.

Heterogeneity of SPECT V/Q acquisition and interpretation across studies is a potential limitation that can cause challenges to be encountered in this review. According to centres and countries, scanning protocols and interpretation criteria vary. An arising challenge is the increasing use of a combined low-dose CT to enable correlation of functional and anatomical lung abnormalities. The use of a combined CT is thought by some experts to further improve the diagnostic performance of SPECT V/Q. Studies will be carefully 
examined for the criteria used for interpretation (positivity threshold) and for the exact imaging protocol.

\section{CONCLUSIONS}

Although SPECT V/Q has already been largely implemented in daily practice, the test is still described as experimental by clinicians. In this systematic review and meta-analysis, objective and widely accepted tools will be used to assess studies' quality. Heterogeneity of studies will be assessed using conventional statistical methods. Accordingly, this systematic review and meta-analysis should provide elements of responses to the ongoing debate about the evidence of SPECT V/Q for the diagnosis of acute PE.

\section{ETHICS AND DISSEMINATION}

Ethical approval and patient consent are not required since this is a systematic review on published studies. The results of this study will be submitted for presentation at relevant national and international conferences, and for publication in a peer-reviewed journal.

Contributors PYLR, PR, GLG and PYS conceived the idea and design for this systematic review. PYLR, PR, AD, MC, GLG and PYS developed the methodology for the systematic review protocol. The contents of this manuscript were drafted by PYLR, GLG and PYS with input from all members of the authorship team. The manuscript was reviewed by PR, CT, AD, MC, HRE and FC for important intellectual content. All authors read and approved the final manuscript.

Funding The authors have not declared a specific grant for this research from any funding agency in the public, commercial or not-for-profit sectors.

Competing interests GLG holds an Early Researcher Award from the Province of Ontario, a CP Has Heart Cardiovascular Clinician Scientist Award from the Heart and Stroke Foundation of Ontario, and the Chair on Diagnosis of Venous Thromboembolism from the Department of Medicine, University of Ottawa.

\section{Patient consent Not required.}

Provenance and peer review Not commissioned; externally peer reviewed. Data sharing statement There is no additional unpublished data from this study. Open Access This is an Open Access article distributed in accordance with the Creative Commons Attribution Non Commercial (CC BY-NC 4.0) license, which permits others to distribute, remix, adapt, build upon this work non-commercially, and license their derivative works on different terms, provided the original work is properly cited and the use is non-commercial. See: http://creativecommons.org/ licenses/by-nc/4.0/

(C) Article author(s) (or their employer(s) unless otherwise stated in the text of the article) 2018. All rights reserved. No commercial use is permitted unless otherwise expressly granted.

\section{REFERENCES}

1. Barritt DW, Jordan SC. Anticoagulant drugs in the treatment of pulmonary embolism. A controlled trial. Lancet 1960;1:1309-12.

2. Carrier M, Le Gal G, Wells PS, et al. Systematic review: case-fatality rates of recurrent venous thromboembolism and major bleeding events among patients treated for venous thromboembolism. Ann Intern Med 2010;152:578-89.

3. Kearon C, Akl EA, Ornelas J, et al. Antithrombotic Therapy for VTE Disease: CHEST Guideline and Expert Panel Report. Chest 2016;149:315-52.

4. Stein PD, Fowler SE, Goodman LR, et al. Multidetector computed tomography for acute pulmonary embolism. N Engl J Med 2006;354:2317-27.
5. Righini M, Le Gal G, Aujesky D, et al. Diagnosis of pulmonary embolism by multidetector CT alone or combined with venous ultrasonography of the leg: a randomised non-inferiority trial. Lancet 2008;371:1343-52.

6. van Belle A, Büller HR, Huisman MV, et al. Effectiveness of managing suspected pulmonary embolism using an algorithm combining clinical probability, D-dimer testing, and computed tomography. JAMA 2006;295:172-9.

7. Le Gal G, Righini M. Controversies in the diagnosis of venous thromboembolism. J Thromb Haemost 2015;13(Suppl 1):S259-65.

8. Anderson DR, Kahn SR, Rodger MA, et al. Computed tomographic pulmonary angiography vs ventilation-perfusion lung scanning in patients with suspected pulmonary embolism: a randomized controlled trial. JAMA 2007;298:2743-53.

9. Konstantinides SV, Torbicki A, Agnelli G, et al. 2014 ESC guidelines on the diagnosis and management of acute pulmonary embolism. Eur Heart J 2014;35:3033-69.

10. PIOPED Investigators. Value of the ventilation/perfusion scan in acute pulmonary embolism. Results of the prospective investigation of pulmonary embolism diagnosis (PIOPED). JAMA 1990;263:2753-9.

11. Perrier A, Desmarais S, Miron MJ, et al. Non-invasive diagnosis of venous thromboembolism in outpatients. Lancet 1999;353:190-5.

12. Wells PS, Ginsberg JS, Anderson DR, et al. Use of a clinical model for safe management of patients with suspected pulmonary embolism. Ann Intern Med 1998;129:997-1005.

13. Salaun PY, Couturaud F, Le Duc-Pennec A, et al. Noninvasive diagnosis of pulmonary embolism. Chest 2011;139:1294-8.

14. Le Roux PY, Robin P, Salaun PY. New developments and future challenges of nuclear medicine and molecular imaging for pulmonary embolism. Thromb Res 2018;163.

15. Roach PJ, Schembri GP, Bailey DL. V/Q scanning using SPECT and SPECT/CT. J Nucl Med 2013;54:1588-96.

16. Mortensen J, Gutte H. SPECT/CT and pulmonary embolism. Eur J Nucl Med Mol Imaging 2014;41(S1):81-90.

17. Bajc M, Neilly JB, Miniati M, et al. EANM guidelines for ventilation/ perfusion scintigraphy : Part 1. Pulmonary imaging with ventilation/ perfusion single photon emission tomography. Eur J Nucl Med Mol Imaging 2009;36:1356-70.

18. Le Roux PY, Pelletier-Galarneau M, De Laroche R, et al. Pulmonary Scintigraphy for the Diagnosis of Acute Pulmonary Embolism: A Survey of Current Practices in Australia, Canada, and France. J Nucl Med 2015;56:1212-7.

19. Phillips JJ, Straiton J, Staff RT. Planar and SPECT ventilation/ perfusion imaging and computed tomography for the diagnosis of pulmonary embolism: A systematic review and meta-analysis of the literature, and cost and dose comparison. Eur J Radiol 2015;84:1392-400.

20. Kan Y, Yuan L, Meeks JK, et al. The accuracy of V/Q SPECT in the diagnosis of pulmonary embolism: a meta-analysis. Acta Radiol 2015:56:565-72.

21. Hess S, Frary EC, Gerke O, et al. State-of-the-Art Imaging in Pulmonary Embolism: Ventilation/Perfusion Single-Photon Emission Computed Tomography versus Computed Tomography Angiography - Controversies, Results, and Recommendations from a Systematic Review. Semin Thromb Hemost 2016;42:833-45.

22. Di Nisio M, van Es N, Büller HR. Deep vein thrombosis and pulmonary embolism. Lancet 2016;388:3060-73.

23. Lapner ST, Kearon C. Diagnosis and management of pulmonary embolism. BMJ 2013;346:f757.

24. Moher D, Shamseer L, Clarke M, et al. Preferred reporting items for systematic review and meta-analysis protocols (PRISMA-P) 2015 statement. Syst Rev 2015;4:1.

25. McGrath TA, Alabousi M, Skidmore B, et al. Recommendations for reporting of systematic reviews and meta-analyses of diagnostic test accuracy: a systematic review. Syst Rev 2017;6:194.

26. Moher D, Liberati A, Tetzlaff J, et al. Preferred reporting items for systematic reviews and meta-analyses: the PRISMA statement. BMJ 2009;339:b2535.

27. Whiting PF, Rutjes AW, Westwood ME, et al. QUADAS-2: a revised tool for the quality assessment of diagnostic accuracy studies. Ann Intern Med 2011;155:529-36.

28. Higgins JP, Altman DG, Gøtzsche PC, et al. The Cochrane Collaboration's tool for assessing risk of bias in randomised trials. BMJ 2011;343:d5928.

29. Sterne JA, Hernán MA, Reeves BC, et al. ROBINS-I: a tool for assessing risk of bias in non-randomised studies of interventions. BMJ 2016;355:i4919.

30. van Beek EJ, Reekers JA, Batchelor DA, et al. Feasibility, safety and clinical utility of angiography in patients with suspected pulmonary embolism. Eur Radiol 1996;6:415-9. 